\title{
Connexin 43 Mediates the Tangential to Radial Migratory Switch in Ventrally Derived Cortical Interneurons
}

\author{
Laura A. B. Elias, ${ }^{1,2}$ Mark Turmaine, ${ }^{3}$ John G. Parnavelas, ${ }^{3}$ and Arnold R. Kriegstein ${ }^{2}$ \\ ${ }^{1}$ Neuroscience Graduate Program and ${ }^{2}$ Institute for Regeneration Medicine, University of California, San Francisco, San Francisco, California 94143, \\ and ${ }^{3}$ Department of Cell and Developmental Biology, University College London, London WC1E 6BT, United Kingdom
}

The adult cerebral cortex is composed of excitatory and inhibitory neurons that arise from progenitor cells in disparate proliferative regions in the developing brain and follow different migratory paths. Excitatory pyramidal neurons originate near the ventricle and migrate radially to their position in the cortical plate along radial glial fibers. On the other hand, inhibitory interneurons arise in the ventral telencephalon and migrate tangentially to enter the developing cortex before migrating radially to reach their correct laminar position. Gap junction adhesion has been shown to play an important mechanistic role in the radial migration of excitatory neurons. We asked whether a similar mechanism governs the tangential or radial migration of inhibitory interneurons. Using short hairpin RNA knockdown of Connexin $43(\mathrm{Cx} 43)$ and $\mathrm{Cx} 26$ together with rescue experiments, we found that gap junctions are dispensable for the tangential migration of interneurons, but that $\mathrm{Cx} 43$ plays a role in the switch from tangential to radial migration that allows interneurons to enter the cortical plate and find their correct laminar position. Moreover this action is dependent on the adhesive properties and the $\mathrm{C}$ terminus of $\mathrm{Cx} 43$ but not the $\mathrm{Cx} 43$ channel. Thus, the radial phase of interneuron migration resembles that of excitatory neuron migration in terms of dependence on $\mathrm{Cx} 43$ adhesion. Furthermore, gap junctions between migrating interneurons and radial processes were observed by electron microscopy. These findings provide mechanistic and structural support for a gap junction-mediated interaction between migrating interneurons and radial glia during the switch from tangential to radial migration.

\section{Introduction}

The adult cerebral cortex is an intricate laminar structure composed of six distinct layers of neurons. The generation of cerebral cortical circuits necessitates two main neuronal classes: glutamatergic excitatory projection neurons and GABAergic inhibitory interneurons that modulate local circuitry. Although excitatory and inhibitory neurons are both generated by radial glial progenitors, they arise from different lineages, and their progenitors are spatially segregated. Thus, these two cell types follow distinct migratory paths from their sites of origin to their eventual positions in the cortical plate.

Excitatory cortical neurons are generated by radial glia in the proliferative regions adjacent to the dorsal wall of the lateral ventricles (Malatesta et al., 2000, 2003; Noctor et al., 2001, 2002). Radial glial cell bodies reside close to the ventricle and send a long radial process to the basement membrane of the pial surface. Newborn excitatory neurons migrate from their birthplace to their final laminar position in the cortical plate in close associa-

Received Nov. 8, 2009; revised March 11, 2010; accepted April 13, 2010.

This work was supported by grants from the National Institutes of Health (to A.R.K.), the California Institute for Regenerative Medicine Graduate Fellowship (to L.A.B.E.), the J. G. Bowes Research Fund, the Wellcome Trust (Grant 074549 to J.G.P.), and an award from the United Kingdom Science and Innovation Network. We are grateful for ideas arising from discussions with members of the Kriegstein Laboratory and G. M. Elias. We thank D. Laird (University of Western Ontario) for (x plasmids, and W. Walantus and J. Agudelo for expert technical support.

Correspondence should be addressed to either Laura A.B. Elias or Arnold R. Kriegstein, Institute for Regeneration Medicine, University of California, San Francisco, 513 Parnassus Avenue, San Francisco, CA 94143. E-mail: Ielias@ stanford.edu or kriegsteina@stemcell.ucsf.edu.

DOI:10.1523/JNEUROSCI.5728-09.2010

Copyright $\odot 2010$ the authors $\quad 0270-6474 / 10 / 307072-06 \$ 15.00 / 0$ tion with radial fibers (Rakic, 1971, 1972, 1988). Although both excitatory and inhibitory neurons reside in increasingly superficial layers in accordance with their birth order, interneurons originate in the ganglionic eminences of the ventral telencephalon and must first migrate tangentially to the neocortex before beginning their radial movement (Miller, 1985; Fairén et al., 1986; Anderson et al., 1997).

Thus the question arises as to whether similar mechanisms govern the tangential and radial migration of cortical interneurons as well as the radial migration of excitatory neurons. A number of molecular mechanisms involved in the radial migration of pyramidal neurons have been found to also play a necessary role in the tangential migration of interneurons, including Doublecortin and p35/Cdk5 (Friocourt et al., 2007; Rakić et al., 2009). However, the different branch dynamics and speed observed during interneuron tangential migration and excitatory neuron radial migration suggests that distinct mechanisms may contribute to these two forms of neuronal migration (Nadarajah and Parnavelas, 2002).

Gap junctions play an important mechanistic role in excitatory neuron radial migration (Nadarajah et al., 1997; Fushiki et al., 2003; Elias et al., 2007; Elias and Kriegstein, 2008; Cina et al., 2009). Gap junctions are large-diameter channels made up of two hemichannels, each composed of six connexin subunits, on opposing membranes that form an aqueous pore between the cytoplasm of adjacent cells (Harris, 2007). The gap junction subunits Connexin 26 (Cx26) and Connexin 43 (Cx43) are highly expressed in the developing cortex and localized to the contact points between radial glial fibers and migrating neurons $(\mathrm{Na}-$ 
darajah et al., 1997; Cina et al., 2007; Elias et al., 2007). Using a short hairpin RNA (shRNA) knockdown strategy, we showed that gap junctions play a necessary role in excitatory neuron radial migration (Elias et al., 2007; Elias and Kriegstein, 2008). This finding is supported by migration studies in the traditional and, more recently, the conditional Cx43 knock-out mouse (Fushiki et al., 2003; Cina et al., 2009). Interestingly, we found that the adhesion between the gap junction hemichannels, but not the pore itself, is necessary for excitatory radial migration (Elias et al., 2007). There is conflicting evidence for the role of the $C$ terminus (CT) of $\mathrm{Cx} 43$ (Cx43CT) in excitatory neuron radial migration, which may relate to the complete or partial deletion of $\mathrm{Cx} 43$ as revisited in the discussion (Elias et al., 2007; Cina et al., 2009).

In this study, we investigate the role of gap junctions in the tangential migration of interneurons from the ganglionic eminences using shRNA knockdown of $\mathrm{Cx} 43$ and Cx26. Furthermore, we use rescue experiments to analyze the mechanistic role of gap junction channels, adhesion, hemichannels, and the CT in the observed phenotypes. Our data suggest that gap junctions are dispensable for tangential migration, but that $\mathrm{Cx} 43$ plays a role in the switch from tangential to radial migration as the interneurons enter the cortical plate. Interestingly, both adhesion and the CT, but not the channel, are necessary to mediate the tangential-toradial switch.

\section{Materials and Methods}

Immunohistochemistry. Immunohistochemistry was performed on embryonic brains subsequent to transcardial perfusion with PBS followed by $4 \%$ paraformaldehyde (PFA) (diluted from 16\% PFA EM grade, Electron Microscopy Sciences 15710) in PBS, and finally, by perfusion with PBS. Brains were immediately dehydrated in $20 \%$ and then $30 \%$ sucrose in PBS, frozen in optimal cutting temperature embedding medium (Tissue-Tec, 4583), and cryostat sectioned at a thickness of $10 \mu \mathrm{m}$. Cx stains were performed less than 1 week after sectioning, or sectioned brains were stored at $-80^{\circ} \mathrm{C}$.

Citrate antigen retrieval was performed for $\mathrm{Cx}$ staining before blocking. Slices were washed with PBS, incubated in citrate buffer $(1.2 \mathrm{~mm}$ citric acid, $8.2 \mathrm{~mm}$ sodium citrate, $\mathrm{pH} 6.0$ with $\mathrm{HCl}$ ) for $5 \mathrm{~min}$ at room temperature (RT), brought to a preboil in the microwave, incubated for $30 \mathrm{~min}$, transferred to citrate buffer at RT, and washed with PBS. Blocking solution contained $2 \%$ gelatin, $10 \%$ serum, and $1 \%$ Triton X-100 in PBS. Sections were incubated overnight in primary antibodies in a 1:5 dilution of blocking solution: rabbit anti-Cx43 (1:50, Zymed) and rabbit anti-Cx26 (1:25, Zymed SI-2800).

Electron microscopy. Litters were removed from anesthetized pregnant Sprague Dawley rats at embryonic days (E; E1, vaginal plug found) 14 $(n=2)$ and $15(n=2)$, decapitated, and their heads immersed in $4 \%$ paraformaldehyde in $0.08 \mathrm{M}$ cacodylate buffer, $\mathrm{pH} 7.2-7.4$, at $4^{\circ} \mathrm{C}$ for 30 min before the brains were removed. The telencephalic vesicles were cut by hand into $1 \mathrm{~mm}$ coronal slices and reimmersed for 3-4 h in cold fixative solution containing $4 \%$ paraformaldehyde and $2.5 \%$ glutaraldehyde in $0.08 \mathrm{M}$ cacodylate buffer, $\mathrm{pH}$ 7.2-7.4. The slices were postfixed in $2 \% \mathrm{OsO}_{4}$ in cacodylate buffer for $2 \mathrm{~h}$, stained in aqueous uranyl acetate for $45 \mathrm{~min}$, dehydrated in ethanol, and embedded in Araldite. Ultrathin sections of silver-gold interference color $(\sim 80 \mathrm{~nm})$ were cut through the dorsal telencephalic wall of the anterior telencephalic vesicle, collected on 200-mesh grids, and examined with a JEOL 1010 electron microscope.

Plasmid constructs. $\mathrm{Cx} 26$ and $\mathrm{Cx} 43$ shRNA hairpins were expressed in the dual promoter construct pLentiLox3.7 (pLLox3.7) (Elias et al., 2007). The following target sequences were used: for Cx26, TCTGGAATTTGCATCCTGCTA; and for Cx43, GCAATTACAACAAGCAAGCTA. Target specificity and knockdown was previously tested (Elias et al., 2007). The rat $\mathrm{Cx} 43$ clone in the $\mathrm{pEYFP-N1}$ vector (Clontech) was a gift from D. Laird (University of Western Ontario, London, ON, Canada). QuikChange site-directed mutagenesis (Stratagene) was used to introduce the various mutations as previously described (Elias et al., 2007). All
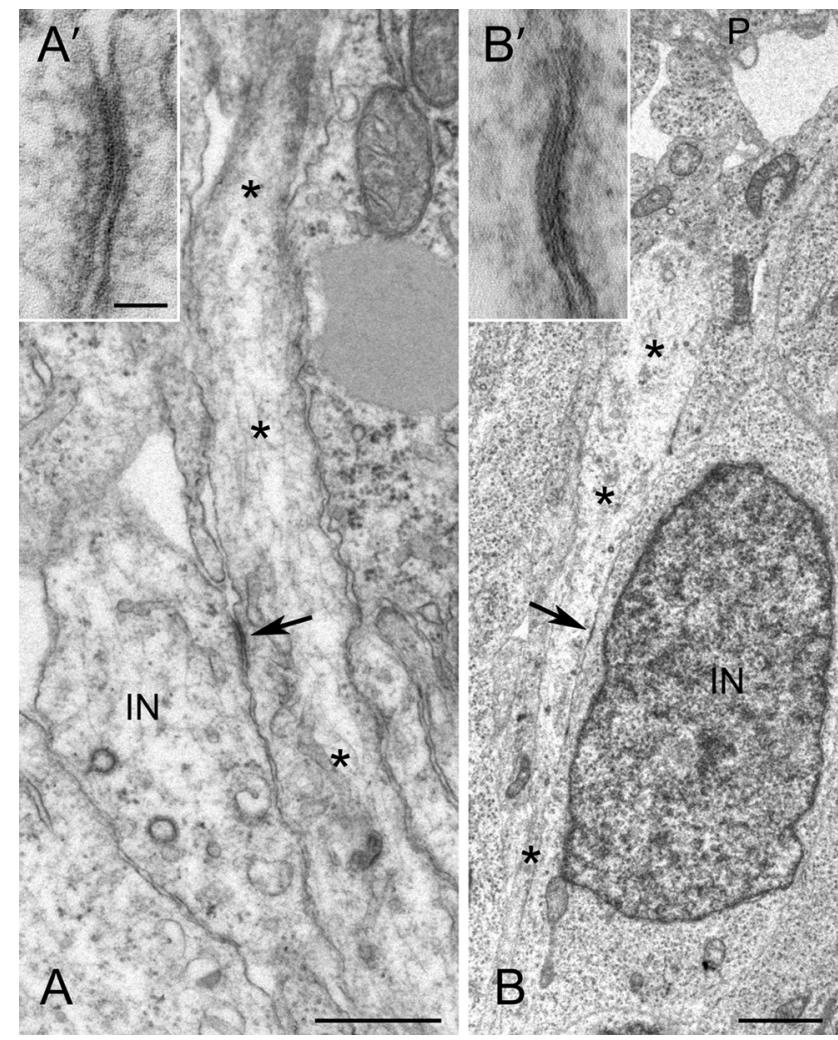

Figure 1. Gap junctions observed by electron microscopy between migrating interneurons and radial glia in the cortical MZ. $A, \boldsymbol{A}^{\prime}$, Gap junction (arrow) between an interneuron process (IN) and radial glial process $\left(^{*}\right) . \boldsymbol{B}, \boldsymbol{B}^{\prime}$, Gap junction (arrow) between an interneuron cell body (IN) and a radial glial process ( $\left.{ }^{*}\right)$. Scale bars: $\boldsymbol{A}, 1 \mu \mathrm{m} ; \boldsymbol{B}, 2 \mu \mathrm{m} ; \boldsymbol{A}^{\prime}, \boldsymbol{B}^{\prime}, 50 \mathrm{~nm}$.

constructs were prepared in an endotoxin-free manner. shRNA constructs were injected at a concentration of $1.9 \mu \mathrm{g} / \mu \mathrm{l}$, and rescue constructs were introduced at a molar ratio of 1:0.4. The Cx43CT was overexpressed at $2.2 \mu \mathrm{g} / \mu \mathrm{l}$ with $0.4 \mu \mathrm{g} / \mu \mathrm{l}$ eYFPN1 control plasmid to enhance visualization.

Slice electroporation and culture. Animals were maintained according to protocols approved by the Institutional Animal Care and Use Committee at University of California, San Francisco. E15.5 rat brains were dissected in ice-cold ACSF bubbled with $5 \% \mathrm{O}_{2} 95 \% \mathrm{CO}_{2}$ and embedded in $4 \%$ low melt agarose (Fisher, BP165-25) in ACSF prewarmed to $50^{\circ} \mathrm{C}$ and subsequently cooled on ice. Vibratome (Leica) sections $(300 \mu \mathrm{m}$ thick) were prepared in ice-cold ACSF bubbled with $5 \% \mathrm{CO}_{2} / 95 \% \mathrm{O}_{2}$. Slices were transferred to RT ACSF and bubbled with 5\% $\mathrm{O}_{2} / 95 \% \mathrm{CO}_{2}$ and then plated on Millicell-CM inserts (Millipore).

A micromanipulator was used to inject DNA directly into the medial ganglionic eminence (MGE) of the slices, and the slices were subsequently electroporated ( $90 \mathrm{~V}, 5 \mathrm{~ms}, 3$ pulses, $500 \mathrm{~ms}$ interval). To shield the slices from the electrical current and direct the electroporation to the MGE, a thin sheet of agarose was placed under the insert, and a small round cylinder of agarose touching only the MGE was placed on the electrode on top of the slice. The insert was then placed in culture medium [25\% HBBS, 66\% Basal Medium Eagle (Invitrogen), 5\% fetal bovine serum, $1 \times \mathrm{Pen} / \mathrm{Strep}, 0.66 \%$ glucose, $1 \% \mathrm{~N} 2$-supplement (Invitrogen)] and stored in a $37^{\circ} \mathrm{C}$ incubator. A full video protocol of the slice culture technique without slice electroporation is available (Elias and Kriegstein, 2007)

Imaging and quantification. Images were collected on an inverted SP5 Spectral Confocal Microscope (Leica). Quantification of cell position and orientation was conducted in a blind manner. For tangential migration, the cortical slice was divided up into five zones as shown (supplemental Fig. 1, available at www.jneurosci.org as supplemental material), and the fraction of cells in each zone was calculated. For orientation, cells in regions III, IV, and V were counted as either radial or tangential 
depending on their orientation with respect to the pial surface of the cortical plate. For laminar position, a grid (width-to-height ratio 1:1.3) with three regions of equal area was fitted to the cortex in region III. The fraction of cells in the upper (from pial surface), middle, and lower one-third of the cortex was quantified for all slices with more than one cell in the grid.

\section{Results}

Gap junctions observed between radial glia and migrating interneurons

Early-born interneurons, derived from the MGE in the ventral telencephalon, migrate to the cortical plate primarily in pathways superficial to the cortical proliferative zones (Anderson et al., 2001). At E15.5 in the rat, we observed the expression of gap junctions including Connexin 26 (Cx26), Cx36, Cx43, and Cx45 in the $\mathrm{MGE}$ as well as in the mantle region where superficial tangentially migrating interneurons are found (supplemental Fig. 1, available at www.jneurosci.org as supplemental material).

We used electron microscopy to determine whether MGE-derived interneurons form gap junctions with radial glia as they migrate into the cortex. Gap junctions with characteristic heptalaminar structure were observed throughout the cortical anlage, especially in the ventricular (VZ) and marginal (MZ) zones at both ages examined. They were most abundant in the VZ where, together with tight junctions and zonula occludens, they made up the specialized junctional complexes often observed between ventricular processes.

We focused our observations to the MZ because interneurons are the only neuronal cell type present in that layer apart from Cajal-Retzius cells, which are highly differentiated at the early stages of corticogenesis (Edmunds and Parnavelas, 1982). Cajal-Retzius cells are characterized by their large size, unique shape, and the presence of a rich complement of organelles in the cytoplasm, most conspicuous of which is the granular endoplasmic reticulum. In contrast, interneurons display a relatively large nucleus and poorly differentiated cytoplasm containing scant organelles. Interneurons, part of the migrating stream of cells that emanate in the ventral telencephalon, first reach the MZ at around E14 (Lavdas et al., 1999), and at this and subsequent stages of corticogenesis they display predominantly horizontal orientation. However, a number of cells appeared to have their elongated cell bodies perpendicular to the pial surface, and are likely those interneurons that have been described to migrate from the MZ toward the cortical plate (Nadarajah and Parnavelas, 2002; Ang et al., 2003; Tanaka et al., 2003).

The majority of gap junctions that we encountered in the MZ were between radial glial processes, identified by their slender, straight, perpendicularly oriented profiles often terminating at the basal lamina of the pial surface. These processes typically showed moderately electron-lucent cytoplasm containing elongated mitochondria, microtubules and enlarged clear vesicles
(Rakic, 1972). The second most frequent type of gap junction in the $\mathrm{MZ}$ was small (typically $80-100 \mathrm{~nm}$ ) and involved neuronal processes and radial glia (Fig. $1 A, A^{\prime}$ ). Neuronal processes, presumably dendrites of interneurons, contained organelles including abundant ribosomes. Also identified in a few fortuitous sections were junctions between cell bodies of interneurons and a radial glia process. In one such example, an interneuron, oriented perpendicular to the pial surface, is in close association and forms a small gap junction (arrow) with a vertical radial glial process ascending toward the pial surface $(\mathrm{P})$ (Fig. $\left.1 B, B^{\prime}\right)$.

\section{Cx43 is necessary for the proper tangential to radial switch of migrating interneurons}

To determine whether gap junctions play a role in interneuronal migration, we manipulated the expression of gap junctions in the MGE by directly electroporating rat organotypic slice cultures at E15.5 with shRNAs that knock down the levels of Cx26 or Cx43 (Elias et al., 2007). We then performed time-lapse microscopy to follow the migration of the electroporated interneurons for $96 \mathrm{~h}$.

In contrast to the effect on the migration of pyramidal neurons (Elias et al., 2007), knockdown of Cx26 or Cx43 did not inhibit the tangential migration of interneurons (supplemental 

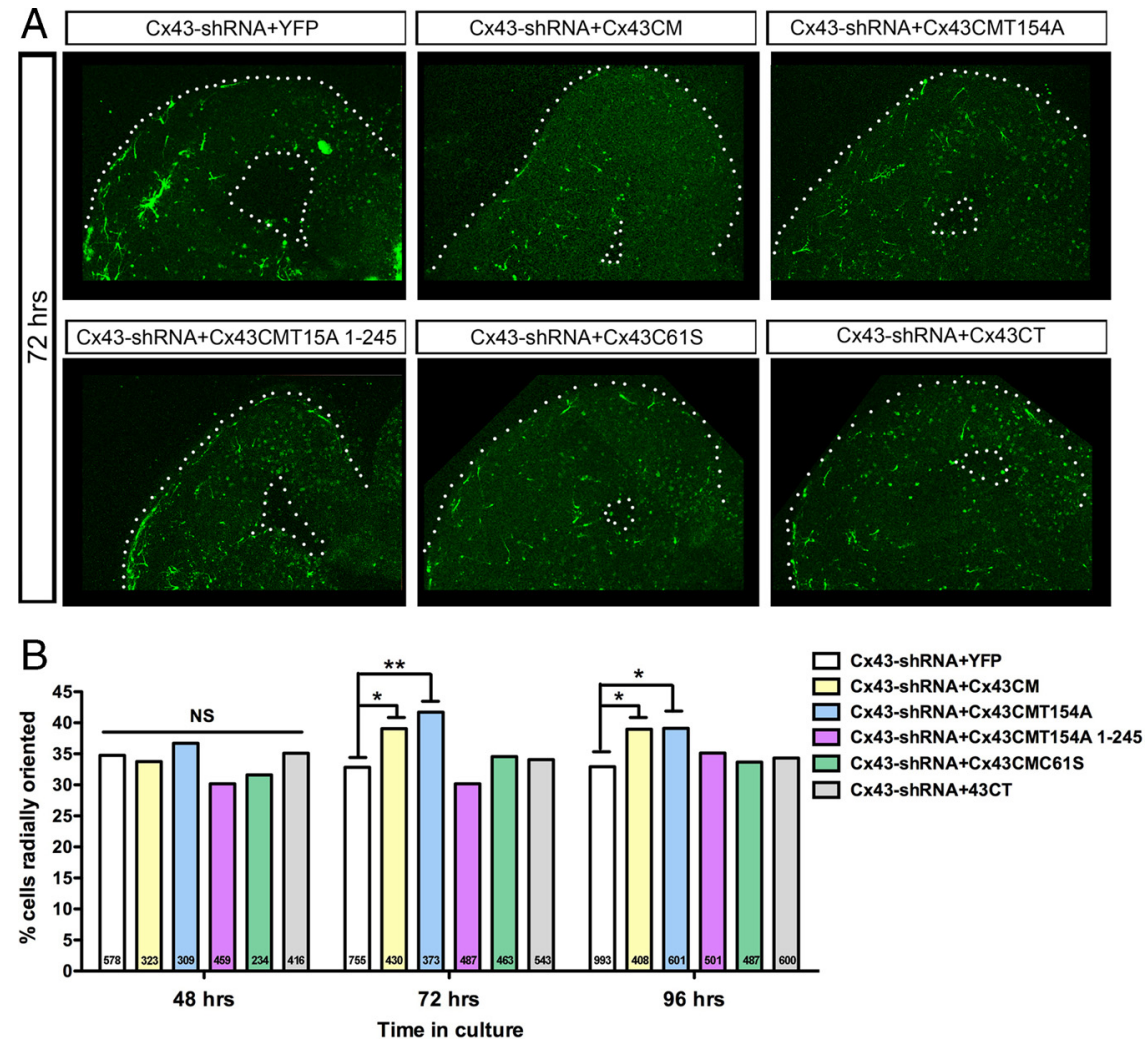

Figure 3. Both $\mathrm{C} \times 43$ adhesion and the $\mathrm{CT}$ are necessary for the switch from tangential to radial migration. $A$, Images of slice cultures at $72 \mathrm{~h}$ after electroporation expressing $\mathrm{Cx} 43$-shRNA along with a $\mathrm{C} 43$ rescue construct. $B$, C $433 \mathrm{CM}$ (function: channel, adhesion, $(\mathrm{T})$ and (x43CMT154A (function: adhesion, $\mathrm{CT}$ ) significantly increased the percentage of radially oriented cells when compared to EYFP at 72 and $96 \mathrm{~h}$ after coelectroporation with $\mathrm{x} \times 43$-shRNA (number of cells per condition indicated on each bar) (Fisher's exact test, $\mathrm{CX} 43 \mathrm{CM}, 72 \mathrm{~h}, p=0.0316,96 \mathrm{~h}, p=0.0358$; (X43CMT154A, $72 \mathrm{~h}, p=0.0045,96 \mathrm{~h}, p=0.0129$ ). CX43CMT154A 1-245 (function: adhesion), CX43CMC61S (function: hemichannel, CT), or Cx43CT (function: CT) did not rescue the orientation defect.

Fig. 2, available at www.jneurosci.org as supplemental material). However, upon closer inspection, we observed that the Cx43-shRNA-expressing interneurons in the neocortex had an altered orientation. Cx43-shRNA-expressing cells had a significantly lower percentage of radial orientation and thus a higher percentage of tangential orientation, suggesting that the $\mathrm{Cx} 43$ shRNA-expressing interneurons have a reduced capacity to switch modes of migration from tangential to radial (Fig. 2A,B). This observation held true at 48,72 , and $96 \mathrm{~h}$ after electroporation (Fig. $2 A, B$ ). Furthermore, the defect in radial orientation in cells expressing Cx43-shRNA resulted in a change in laminar positioning, suggesting that the misoriented cells are impaired in their ability to migrate radially. While laminar positioning was normal $48 \mathrm{~h}$ after electroporation, by $96 \mathrm{~h}$ significantly more Cx43-shRNA cells were localized in the upper one-third of the cortex near the pial surface in comparison to control or Cx26shRNA-expressing cells (Fig. $2 A, B$ ). Interestingly, this is the first manipulation, to our knowledge, that clearly inhibits only the radial and not the tangential mode of interneuronal migration, suggesting that these two modes of migration are molecularly distinct.

\section{Cx43 adhesion and CT are necessary to rescue the orientation defect}

There are a number of mechanisms by which gap junctions might mediate migration, including the chemical coupling of cells, extracellular release of substances through hemichannels, adhesion between cells, or signaling through the intracellular CT. Our previous work studying the role of $\mathrm{Cx} 26$ and $\mathrm{Cx} 43$ in the radial migration of excitatory neurons suggests that the adhesive properties of gap junctions but not the channel or the CT are necessary to mediate migration (Elias et al., 2007; Elias and Kriegstein, 2008). We performed a similar set of selective rescue experiments in this study to determine the mechanism of gap junction action in the tangential to radial switch in interneuron migration. Rescue constructs, or enhanced yellow fluorescent protein (EYFP) alone, were electroporated with the $\mathrm{Cx} 43$-shRNA construct at a set molar ratio. The tangential to radial switch was significantly rescued at $72 \mathrm{~h}$ by the expression of $\mathrm{Cx} 43$ with conservative mutations that prevent targeting by the shRNA (Cx43CM) (Elias et al., 2007) (Fig. $3 A, B)$. This suggests that the phenotype is specific to Cx43 knockdown and is not an off-target effect of shRNA expression.

To determine whether the channel is necessary, a conserved tyrosine in the third transmembrane domain of $\mathrm{Cx} 43$ was mutated (Cx43CMT154A) (Elias et al., 2007). These dominant-negative connexin mutants are able to make adhesions but have a closed channel and thus are not able to mediate exchange between cells or with the extracellular environment (Beahm et al., 2006; Elias et al., 2007). Interestingly Cx43CMT154A significantly rescues the tangential to radial switch at $72 \mathrm{~h}$, suggesting that the channel function is not necessary (Fig. $3 A, B)$. To determine whether adhesion is necessary, one of the conserved extracellular cysteines was mutated (Cx43CMC61S) such that the $\mathrm{Cx}$ subunit is unable to form gap junctions but maintains the ability to form functional hemichannels (Lin et al., 2002; Elias et al., 2007). Expression of Cx43CMC61S was not able to rescue the switch, suggesting that adhesion is necessary (Fig. $3 A, B$ ).

Next we sought to determine whether the Cx43CT played a necessary role in mediating the tangential to radial migratory switch. With respect to excitatory neuron radial migration, deletion of the CT before or after the microtubule binding domain (1-238 or 1-245) did not disrupt the ability of Cx43CMT154A to rescue the migration defect (Elias et al., 2007). In contrast, Cx43CMT154A 1-245 was unable to rescue the switch from tangential to radial orientation, suggesting a role for the CT in this aspect of interneuronal migration (Fig. $3 A, B$ ). In addition, $\mathrm{Cx} 43 \mathrm{CT}$ alone is not sufficient to rescue the switch suggesting that both gap junction adhesion and the CT are necessary.

We hypothesized that if a signaling interaction on the Cx43CT was important for mediating the tangential to radial orientation switch, we might be able to inhibit the switch by flooding the system with excess Cx43CT. Interestingly, overexpression of the $\mathrm{Cx} 43 \mathrm{CT}$ alone acts as an apparent dominant-negative inhibiting the migratory switch (Fig. $4 A, B$ ).

In accordance with our previous study on excitatory neurons (Elias et al., 2007; Elias and Kriegstein, 2008), we observed an 
A

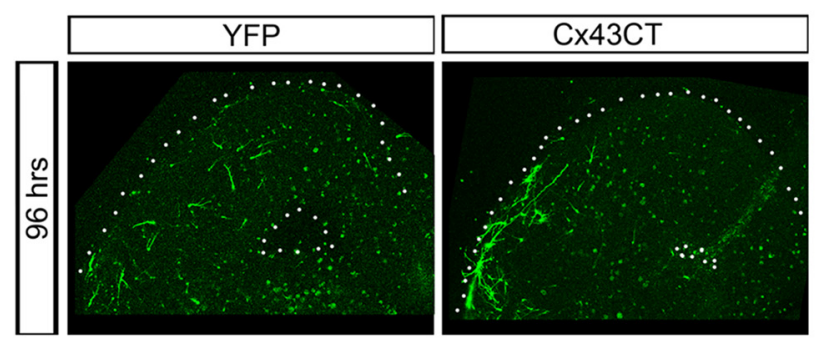

B

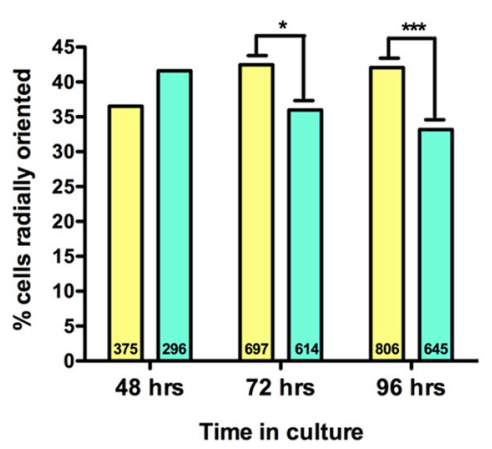

C
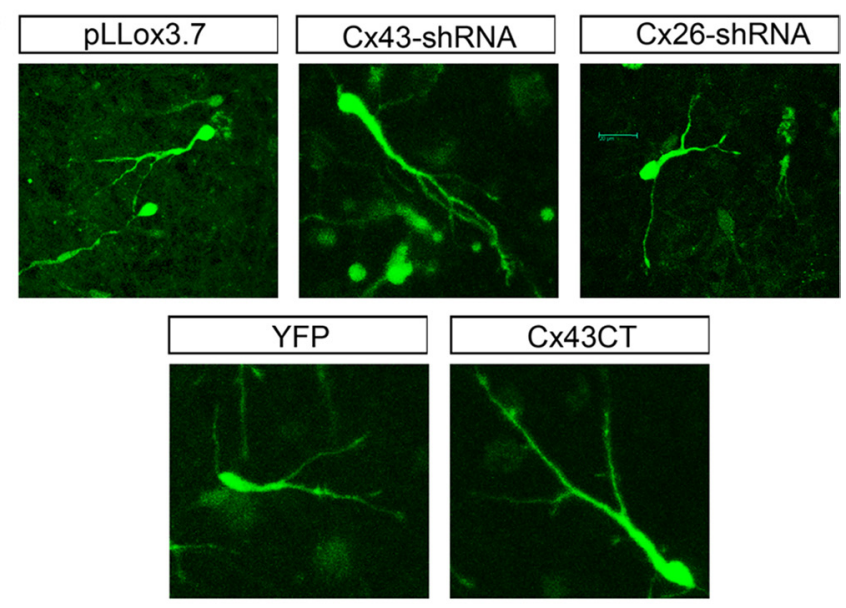

D
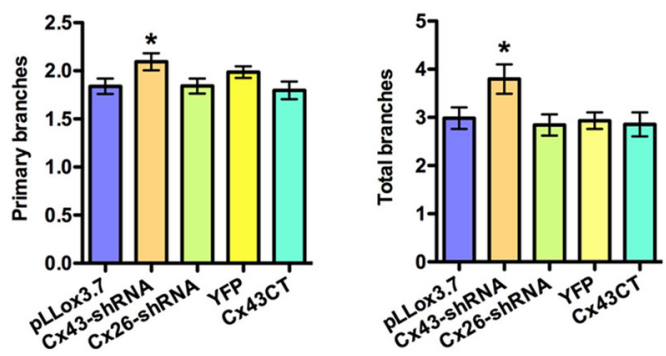

Figure 4. Overexpressing the $\mathrm{Cx} 43 \mathrm{CT}$ alone has a dominant-negative effect, thus inhibiting the tangential to radial switch at 72 and $96 \mathrm{~h}$, but does not affect cell branching patterns. $A$, Images of slice cultures $96 \mathrm{~h}$ after electroporation with EYFP or Cx43CT + EYFP. B, Compared to EYFP, Cx43CT significantly decreases the percentage of radially oriented cells at 72 and $96 \mathrm{~h}$ (number of cells per condition indicated on each bar) (Fisher's exact test, $72 \mathrm{~h}, p=0.0174 ; 96 \mathrm{~h}, p=0.0006$ ) (left). At $96 \mathrm{~h}$, compared to EYFP, there is a developing trend for (x43CT expression to increase the fraction of cells found in the outer one-third of the cortex ( $t$ test, $p=0.1429$ ) (right). C, Images of migrating interneurons in the dorsal cortex at $72 \mathrm{~h}$ after electroporation. $D$, Cx43-shRNA-expressing cells display a significantly higher number of primary and total branches ( $t$ test, pLLox3.7 vs (x43-shRNA, primary, $p=0.0349$; total, $p=0.0340$ ). Cx26-shRNA and the Cx43CT do not affect branching patterns.

increase in primary branches and total branches in migrating interneurons expressing Cx43-shRNA (Fig. 4C,D). We found no change in branching in Cx26-shRNA-expressing or Cx43CToverexpressing cells (Fig. $4 C, D$ ). The lack of increased branching in $\mathrm{Cx} 43 \mathrm{CT}$-overexpressing cells suggests that the Cx43CT plays a role in turning that is independent of the overbranching phenotype seen when $\mathrm{Cx} 43$ is downregulated in both interneurons and excitatory neurons.

In sum, our findings suggest that $\mathrm{Cx} 43$ acts to promote a change in the orientation of migrating interneurons from tangential to radial, thereby allowing them to invade the cortical plate and find their correct laminar location. Furthermore, both the adhesive properties of $\mathrm{Cx} 43$ and the CT are necessary for this action.

\section{Discussion}

Our studies suggest that there are important mechanistic differences between the role of gap junctions in the radial migration of excitatory pyramidal neurons and the tangential and radial migration of inhibitory neurons in the neocortex. During radial migration of pyramidal neurons, both $\mathrm{Cx} 43$ and $\mathrm{Cx} 26$ play an adhesive role in mediating migration along radial fibers (Elias et al., 2007). On the other hand, Cx26-shRNA does not effect the tangential migration of interneurons or the tangential to radial switch as interneurons enter the neocortex. While Cx43-shRNA does not inhibit the tangential migration of interneurons, it does inhibit the tangential to radial orientation switch. The role of $\mathrm{Cx} 43$ in interneuron radial orientation appears to be dependent on the adhesive but not the channel properties of $\mathrm{Cx} 43$.
Unlike our results in excitatory neuron migration suggesting that the $\mathrm{Cx} 43 \mathrm{CT}$ is not necessary for radial migration (Elias et al., 2007), the Cx43CT is necessary to rescue the interneuron orientation defect. This suggests that an additional signaling component residing within the CT triggers the change in orientation. $\mathrm{Cx} 43$ has a long CT that interacts with a variety of proteins, including v-Src, c-Src, Zona Occludens-1, and tubulin (Giepmans and Moolenaar, 1998; Giepmans et al., 2001; Lin et al., 2001; Giepmans, 2004). Interestingly, when Cx43CT is overexpressed it plays a dominant-negative role and inhibits the tangential-toradial switch, suggesting that it is occluding the endogenous signaling pathway. The CT truncation mutant used for rescue experiments in this study left the tubulin binding domain intact, excluding the possibility of its importance; a signaling interaction or modification downstream of the tubulin binding site must be critical for the tangential to radial switch. Furthermore, the necessity of the Cx43CT for interneuron radial orientation may explain why Cx26 plays a role in excitatory neuron but not interneuron radial migration as $\mathrm{Cx} 26$ has a relatively very short $\mathrm{CT}$ with very limited interactions.

Thus in our selective rescue experiments, we find that the $\mathrm{Cx} 43 \mathrm{CT}$ is necessary to rescue the defect in the tangential to radial orientation switch in Cx43-shRNA-expressing interneurons. However, we find that the CT of $\mathrm{Cx} 26$ or $\mathrm{Cx} 43$ is not necessary to rescue the defect in the radial migration of excitatory neurons expressing Cx26-shRNA or Cx43-shRNA (Elias et al., 2007). In contrast to our results, Cina et al. (2009) suggest that the Cx43CT is necessary for excitatory neuron radial migration using a con- 
ditional genetic knock-out and rescue strategy as well as transgenic mice expressing a $\mathrm{Cx} 43$ lacking the CT. While it is difficult to know the exact reason for the discrepancy in our results with regards to excitatory neuronal migration, we suggest that it may center on the global versus limited manipulation of $\mathrm{Cx} 43$. In our shRNA electroporation studies, a small subset of the neurons and radial glia are targeted. In contrast, the genetic techniques used by Cina et al. (2009) target the cortex as a whole. In this regard we suggest that the Cx43CT may not be necessary in migrating excitatory neurons, as we are able to rescue their migration by expressing Cx43-shRNA with Cx43CMT54A 1-238 or Cx43CMT154A 1-245. However, these neurons may be migrating on radial glia that express wild-type Cx43. In Cina et al. (2009), both the neurons and the radial glia only express the form of $\mathrm{Cx} 43$ lacking the CT. Thus the Cx43CT may not be necessary in the excitatory neurons but may be necessary in the radial glia to support neuronal migration. Further studies may clarify some of these issues.

There are a number of important implications of the role of $\mathrm{Cx} 43$ in the tangential to radial switch in interneuron migration. First, this suggests that the mechanism of tangential migration is distinct from that of radial migration and that gap junctions play a more prominent role radial migration. Second, the gap junctions observed between interneurons and radial fibers by electron microscopy coupled with the adhesive mechanistic role of $\mathrm{Cx} 43$ suggest that interneurons interact with radial glia during the radial aspect of migration. A number of groups have suggested that radial glia are important for the migration of interneurons by observing the morphology as well as the movements of interneurons in contact with radial glia and by ablating radial glia (Polleux et al., 2002; Poluch and Juliano, 2007; Yokota et al., 2007). The data presented here provide the first structural and mechanistic support for an interaction between interneurons and radial glia during the switch from tangential to radial migration. Thus, we suggest that $\mathrm{Cx} 43$ controls the radial migration of both interneurons and excitatory neurons through adhesive interactions with radial glial fibers. Further studies will help determine other mechanisms that coordinate the migration of interneurons and excitatory neurons.

\section{References}

Anderson SA, Eisenstat DD, Shi L, Rubenstein JL (1997) Interneuron migration from basal forebrain to neocortex: dependence on Dlx genes. Science 278:474-476.

Anderson SA, Marín O, Horn C, Jennings K, Rubenstein JL (2001) Distinct cortical migrations from the medial and lateral ganglionic eminences. Development 128:353-363.

Ang ES Jr, Haydar TF, Gluncic V, Rakic P (2003) Four-dimensional migratory coordinates of GABAergic interneurons in the developing mouse cortex. J Neurosci 23:5805-5815.

Beahm DL, Oshima A, Gaietta GM, Hand GM, Smock AE, Zucker SN, Toloue MM, Chandrasekhar A, Nicholson BJ, Sosinsky GE (2006) Mutation of a conserved threonine in the third transmembrane helix of alpha- and beta-connexins creates a dominant-negative closed gap junction channel. J Biol Chem 281:7994-8009.

Cina C, Bechberger JF, Ozog MA, Naus CC (2007) Expression of connexins in embryonic mouse neocortical development. J Comp Neurol 504:298-313.

Cina C, Maass K, Theis M, Willecke K, Bechberger JF, Naus CC (2009) Involvement of the cytoplasmic C-terminal domain of connexin 43 in neuronal migration. J Neurosci 29:2009-2021.

Edmunds SM, Parnavelas JG (1982) Retzius-Cajal cells: an ultrastructural study in the developing visual cortex of the rat. J Neurocytol 11:427-446.

Elias L, Kriegstein A (2007) Organotypic slice culture of E18 rat brains. J Vis Exp:235.

Elias LA, Kriegstein AR (2008) Gap junctions: multifaceted regulators of embryonic cortical development. Trends Neurosci 31:243-250.
Elias LA, Wang DD, Kriegstein AR (2007) Gap junction adhesion is necessary for radial migration in the neocortex. Nature 448:901-907.

Fairén A, Cobas A, Fonseca M (1986) Times of generation of glutamic acid decarboxylase immunoreactive neurons in mouse somatosensory cortex. J Comp Neurol 251:67-83.

Friocourt G, Liu JS, Antypa M, Rakic S, Walsh CA, Parnavelas JG (2007) Both doublecortin and doublecortin-like kinase play a role in cortical interneuron migration. J Neurosci 27:3875-3883.

Fushiki S, Perez Velazquez JL, Zhang L, Bechberger JF, Carlen PL, Naus CC (2003) Changes in neuronal migration in neocortex of connexin 43 null mutant mice. J Neuropathol Exp Neurol 62:304-314.

Giepmans BN (2004) Gap junctions and connexin-interacting proteins. Cardiovasc Res 62:233-245.

Giepmans BN, Moolenaar WH (1998) The gap junction protein connexin43 interacts with the second PDZ domain of the zona occludens-1 protein. Curr Biol 8:931-934.

Giepmans BN, Verlaan I, Hengeveld T, Janssen H, Calafat J, Falk MM, Moolenaar WH (2001) Gap junction protein connexin-43 interacts directly with microtubules. Curr Biol 11:1364-1368.

Harris AL (2007) Connexin channel permeability to cytoplasmic molecules. Prog Biophys Mol Biol 94:120-143.

Lavdas AA, Grigoriou M, Pachnis V, Parnavelas JG (1999) The medial ganglionic eminence gives rise to a population of early neurons in the developing cerebral cortex. J Neurosci 19:7881-7888.

Lin JH, Takano T, Cotrina ML, Arcuino G, Kang J, Liu S, Gao Q, Jiang L, Li F, Lichtenberg-Frate H, Haubrich S, Willecke K, Goldman SA, Nedergaard M (2002) Connexin 43 enhances the adhesivity and mediates the invasion of malignant glioma cells. J Neurosci 22:4302-4311.

Lin R, Warn-Cramer BJ, Kurata WE, Lau AF (2001) v-Src phosphorylation of connexin 43 on Tyr247 and Tyr265 disrupts gap junctional communication. J Cell Biol 154:815-827.

Malatesta P, Hartfuss E, Götz M (2000) Isolation of radial glial cells by fluorescent-activated cell sorting reveals a neuronal lineage. Development 127:5253-5263.

Malatesta P, Hack MA, Hartfuss E, Kettenmann H, Klinkert W, Kirchhoff F, Götz M (2003) Neuronal or glial progeny: regional differences in radial glia fate. Neuron 37:751-764.

Miller MW (1985) Cogeneration of retrogradely labeled corticocortical projection and GABA-immunoreactive local circuit neurons in cerebral cortex. Brain Res 355:187-192.

Nadarajah B, Parnavelas JG (2002) Modes of neuronal migration in the developing cerebral cortex. Nat Rev Neurosci 3:423-432.

Nadarajah B, Jones AM, Evans WH, Parnavelas JG (1997) Differential expression of connexins during neocortical development and neuronal circuit formation. J Neurosci 17:3096-3111.

Noctor SC, Flint AC, Weissman TA, Dammerman RS, Kriegstein AR (2001) Neurons derived from radial glial cells establish radial units in neocortex. Nature 409:714-720.

Noctor SC, Flint AC, Weissman TA, Wong WS, Clinton BK, Kriegstein AR (2002) Dividing precursor cells of the embryonic cortical ventricular zone have morphological and molecular characteristics of radial glia. J Neurosci 22:3161-3173.

Polleux F, Whitford KL, Dijkhuizen PA, Vitalis T, Ghosh A (2002) Control of cortical interneuron migration by neurotrophins and PI3-kinase signaling. Development 129:3147-3160.

Poluch S, Juliano SL (2007) A normal radial glial scaffold is necessary for migration of interneurons during neocortical development. Glia 55:822-830.

Rakic P (1971) Guidance of neurons migrating to the fetal monkey neocortex. Brain Res 33:471-476.

Rakic P (1972) Mode of cell migration to the superficial layers of fetal monkey neocortex. J Comp Neurol 145:61-83.

Rakic P (1988) Specification of cerebral cortical areas. Science 241:170-176.

Rakić S, Yanagawa Y, Obata K, Faux C, Parnavelas JG, Nikolić M (2009) Cortical interneurons require $\mathrm{p} 35 / \mathrm{Cdk} 5$ for their migration and laminar organization. Cereb Cortex 19:1857-1869.

Tanaka D, Nakaya Y, Yanagawa Y, Obata K, Murakami F (2003) Multimodal tangential migration of neocortical GABAergic neurons independent of GPI-anchored proteins. Development 130:5803-5813.

Yokota Y, Gashghaei HT, Han C, Watson H, Campbell KJ, Anton ES (2007) Radial glial dependent and independent dynamics of interneuronal migration in the developing cerebral cortex. PLoS ONE 2:e794. 